\title{
A Systematic Review on Percutaneous Fixation of Anterior Column Fractures of the Acetabulum
}

\author{
M.A.Ashour, M.A.Mselhy, S.A.Shoulah and M.S.Abdelhamed \\ Orthopedic Surgery Dept., Faculty of Medicine, Benha Univ., Benha, Egypt \\ E-Mail:Abdelhamed6@gmail.com
}

\begin{abstract}
The current standard treatment of anterior column acetabular fractures includes formal open reduction with internal fixation (ORIF) through a variety of anterior approaches. Nonetheless, extensile introduction can prompt entanglements. Percutaneous negligibly obtrusive careful adjustment for a front section break of hip bone socket has become an acknowledged treatment strategy for as far back as quite a long while. The reason for this investigation is to give combined information about signs and results of percutaneous obsession of cracks of foremost segment of hip bone socket. This orderly survey comprised of 4 stages, including a methodical inquiry of the writing by utilizing PubMed and The Cochrane Library (Step1), determination of clinical investigations in the English language bound to cracks of hip bone socket which went through percutaneous obsession of foremost section (Step 2), study qualities were deliberately removed from the chosen papers (Step 3), and extraction of information on clinical results (Step 4). The inquiry yielded 14 papers qualified for our investigation. $68 \%$ were guys and $32 \%$ were females. The mean age of the patients was 52 yr. The primary system of injury is RTA $59 \%$. The mean employable time was $43.4 \mathrm{~min}$ and the mean blood misfortune was $54.3 \mathrm{ml}$. Just 25 patients $(5.8 \%)$ had careful inconveniences. The majority of articles empower Partial weight-holding on for ROM practices in the first post-usable day. The mean HHS was 87.7 (acceptable). Post-usable radiological result was anatomical in $72 \%$ though radiological result in last visit was Excellent in $63 \%$ and Good in 20\%. PCS obsession of breaks of the foremost segment of the hip bone socket with either traditional or mechanized route framework fluoroscopy could be applied not exclusively to non-uprooted cracks yet in addition to dislodged breaks amiable to shut or restricted open decrease. The mechanized route framework is better as it gives a multi-planar perspectives which give a $3 \mathrm{D}$ creative mind of the life structures of the pelvis, diminishing the quantity of pictures taken and this will diminish fluoroscopic time introduction and blood misfortune. Contrasted with ORIF, PCS obsession accomplishes a comparative nature of decrease and utilitarian result yet is related with less complexities, littler intraoperative blood misfortune, shorter usable time and permit early weight-bearing and recovery.
\end{abstract}

\section{Introduction}

The occurrence of acetabular cracks has been generally steady throughout the long term, with 37 pelvic breaks for each 100,000 yearly and just $10 \%$ of these including the hip bone socket [1].

Anyway the occurrence of acetabular cracks in older patients is expanding presumably because of the mix of more prominent life span with a more dynamic way of life and a general diminishing in the rate of liquor related injury in more youthful grown-ups [2].

The majority of the acetabular cracks result from high-energy injury like engine vehicle mishaps or tumbles from a noteworthy stature. They are commonly considered as genuine wounds and connected with other musculoskeletal, head and instinctive wounds. Interestingly, delicacy acetabular cracks in osteoporotic patients can be brought about by straightforward lowenergy tumbles from standing tallness [3].

Acetabular breaks were dealt with non-carefully until the 1960s, when Judet and Letournel depicted the hip bone socket as an altered $\mathrm{Y}$-arrangement shaped by front and back segments of bone, which join in the supraacetabular area. The foremost and back dividers reach out from each particular section and structure the cup of the hip bone socket. The foremost and back segments interface with the pivotal skeleton through a swagger of bone called the sciatic support [4].

This depiction has permitted specialists to order crack hip bone socket as straightforward and complex ones, in the interim it grows the perspective on the hip bone socket as a 3-dimensional structure, zeroing in on obsession of every section as an autonomous entity [5].

Eventually, the objective of treatment is to accomplish anatomical decrease as even the smallest imperfection $(<3 \mathrm{~mm})$ in the articular surface can prompt post-horrendous joint pain and poor practical results [6]

The stretch among break and medical procedure influences crack decrease where careful therapy following 11 days is more troublesome because of delicate tissue contracture, association of the blood coagulation and callus development additionally The sort of break influences the chance of sufficient anatomical decrease. Osteopaenic bone and progressed age increment the danger of insufficient decrease and obsession of cracks [7].

In this manner, open anatomical decrease of the articular surface joined with unbending inward

obsession turned into the standard strategy in the treatment of most uprooted breaks as anatomic decrease is very diffcult to acquire by shut methods, additionally standard plate and screw obsession develops, which require open medical procedure, have been demonstrated to be more grounded than their percutaneous partners, showing more prominent yield quality and maximal burden at disappointment $[6,8]$.

Nonetheless, open decrease can cause grimness, including neurovascular injury, blood misfortune, heterotopic bone arrangement, wound contamination and issues with wound recuperating [9].

The percutaneous methodology is related with less 
complexities than open strategies, however acetabular calculation makes percutaneous screw addition a difficult strategy [10].

The percutaneous method is suggested for nondisplaced or marginally dislodged cracks, large, osteoporotic and older patients where not exactly anatomic decrease is acknowledged [3,11].

\section{Aim of the work}

The purpose of this study is to provide cumulative data about indications and outcomes of percutaneous fixation of fractures of anterior column of acetabulum.

\section{Patients and methods}

This deliberate survey comprised of 4 stages, including a methodical inquiry of the writing (Step 1), choice of studies (Step 2), recording of study attributes (Step 3), and extraction of information on clinical results and their examinations between various careful gatherings (Step 4).

Stage 1: information sources and search methodology

The writing search was finished utilizing the accompanying electronic information bases: PubMed, SCOPUS, Web of Science, and The Cochrane Library. The hunt systems were created and altered by the creators to expand the affectability. The hunt methodology incorporated a few distinct terms and equivalent words for acetabular crack and pelvic break in blend with, careful administration, insignificantly obtrusive obsession and radiological introduction, blood misfortune, utilitarian capacity or physical movement.

Stage 2: choice of studies and Screening of titles and modified works.

To start with, all titles and digests were screened for the accompanying models: Article concerned (1) a clinical report. Additionally, (2) just articles in the English language were considered for incorporation in this audit. For every chose title and modified works, the full-text articles were accumulated for additional screening. Screening of full-text papers incorporated the initial two measures enhanced with the accompanying models: the examination was (3) kept to cracks of hip bone socket which went through percutaneous obsession of foremost segment. Also, the reference records in the chose papers were examined for important examinations.

Stage 3: study attributes

The accompanying investigation attributes were deliberately separated from the chosen full-text papers: creator, year of distribution, mediation types (percutaneous obsession of front acetabular section), study configuration (Randomized controlled preliminaries, or Clinical preliminaries Phase II), the quantity of patients in percutaneos obsession, age and term of the development (mean or middle and range), clinical and radiological result.

Stage 4: results of the included investigations

The accompanying result attributes and scores were deliberately separated from the chosen full-text papers: activity time, fluoroscopy introduction time, obsession method, blood misfortune during activity, difficulties, hip scoring for result and postoperative weight-bearing.

\section{Results}

Initially, our search yielded 86 publications. After screening of their titles and abstracts for removal of the duplicates and publications in language other than English, 38 articles remained. After full text review of the remaining articles, case reports and reviews and studies not describing outcomes were excluded leaving 14 papers eligible for our study.

Table (1) Demographic Characteristics.

\begin{tabular}{lccccc}
\hline Authors & Year & No of patients & $\begin{array}{c}\text { Sex } \\
\text { Male/Female }\end{array}$ & Mean age yr & $\begin{array}{c}\text { Mechanism } \\
\text { RTA/FALL }\end{array}$ \\
\hline Crowl [12] & 2002 & 23 & N/A & 43 & $16 / 7$ \\
Moushine [13] & 2005 & 21 & $12 / 9$ & 81 & $7 / 14$ \\
Lin [14] & 2007 & 3 & $2 / 1$ & 39 & N/A \\
Frank [15] & 2010 & 10 & $6 / 4$ & 46 & N/A \\
Hong [16] & 2010 & 18 & $14 / 4$ & 42.1 & N/A \\
Gary [17] & 2012 & 35 & $25 / 10$ & 70 & $18 / 10$ \\
Kazemi [18] & 2012 & 28 & $21 / 7$ & 49 & $7 / 5$ \\
Schwabe [19] & 2014 & 12 & $9 / 3$ & 65 & $17 / 5$ \\
He [20] & 2016 & 22 & $15 / 7$ & 35.6 & $12 / 4$ \\
Zhang [21] & 2016 & 16 & $11 / 5$ & 38.8 & $87 / 56$ \\
Qoreishi [22] & 2018 & 143 & $95 / 48$ & 29 & $12 / 8$ \\
El-ashhab [23] & 2019 & 20 & $17 / 3$ & 86 & 0.1 \\
Wong [24] & 2019 & 17 & $1 / 16$ & 74 & N/A \\
Ernstberger [25] & 2020 & 62 & $50 / 12$ & Mean age & $189 / 131$ \\
Total & & 430 & $278 / 129$ & 52 yr & $59 \% / 41 \%$ \\
\hline
\end{tabular}

The exploration revieled 14 papers, distributed from 2002 to 2020 including 430 patients. Of these, 278(68\%) were guys and $129(32 \%)$ were females. The mean age of the patients was $52 \mathrm{yr}$ extending from ( $29 \mathrm{yr}$ to $81 \mathrm{yr}$ ).
The fundamental instrument of injury is RTA $59 \%$ while it is $41 \%$ by tumbling from a stature. Table (1)

Intraoperative, 6 examinations went through percutaneous obsession utilizing traditional (C-arm) 
fluoroscopy, 4 investigations utilized mechanized route framework and 2 investigations utilized both. The mean fluoroscopic presentation time was $102.2 \mathrm{~s}$ for regular fluoroscopy however it was $34.7 \mathrm{~s}$ for automated route framework. The mean usable time was $43.4 \mathrm{~min}$ extending from (24.6 min to $94 \mathrm{~min}$ ). In our examination around 61 breaks of 167 cracks required shut decrease while another 34 breaks required further open decrease through a small open methodology however there were
72 breaks not needing decrease procedures. For obsession either $6.5 \mathrm{~mm}$ or $7.3 \mathrm{~mm}$ halfway strung screw were utilized through a retrograde or antegrade or retrograde strategy for inclusion of a guide wire then addition of the screw by the antegrade method.

The mean pre-employable crack removal was 5.9 $\mathrm{mm}$ which became $1.8 \mathrm{~mm}$ after break decrease and obsession, additionally the mean pre-usable break step was 2.2 which become $0.9 \mathrm{~mm}$ post-operative Table (2).

Table (2) Display of fracture gap and step.

\begin{tabular}{lcccc}
\hline \multirow{2}{*}{ Authors } & \multicolumn{2}{c}{ Fracture displacement(gap) /mm } & \multicolumn{2}{c}{ Fracture step/mm } \\
\cline { 2 - 4 } & Pre-op & Post-op & Pre-op & Post-op \\
\hline Crowl & 8.9 & 2.3 & N/A & N/A \\
Frank & N/A & 1.3 & N/A & N/A \\
Hong & 10 & 3 & 4 & 2 \\
Kazemi & 3.9 & 2.8 & N/A & N/A \\
Schawbe & 4.1 & 0.4 & 1.2 & 0.2 \\
Ernstberger & 2.5 & 1.2 & 1.3 & 0.5 \\
Results (Mean) & $5.9 \mathrm{~mm}$ & $1.8 \mathrm{~mm}$ & $2.2 \mathrm{~mm}$ & $0.9 \mathrm{~mm}$ \\
\hline
\end{tabular}

The normal blood misfortune was $54.3 \mathrm{ml}$ extending from 10 to $94 \mathrm{ml}$, where in customary fluoroscopy bunch blood misfortune was $57 \mathrm{ml}$ yet in modernized route bunch blood misfortune was $42 \mathrm{ml}$.

In around 430 patients remembered for our examination, just 25 patients $(5.8 \%)$ had careful inconveniences where 2 patients had post-employable profound careful diseases, 1 patient had transient femoral paralysis, 5 patients had intraoperative screw infiltration, 8 patients had screw back-out, 5 patients had Loss of decrease, one patient had break non-association and 3 Unkown complexities.

The majority of articles in our investigation support Partial weight-holding on for ROM practices in the first post-employable day and permit full weight bearing in a scope of 4 to about two months yet one article (Kazemi et al) empower quick post-usable full weight bearing. The mean span for crack recuperating after break obsession was 9.8 weeks.

The useful result was surveyed either by Merle d'Aubigne'scale or HHS. As indicated by Merle $40.2 \%$ of patients had brilliant result, $46 \%$ great, $5.9 \%$ reasonable and $7.9 \%$ poor. The mean HHS was 87.7 (acceptable).

The radiological result was evaluated by matta measures either postoperative or last visit of development:

Post-employable radiological result was anatomical in $72 \%$, Imperfect in $24 \%$ and Poor in $4 \%$ of the patients.

Whereas Radiological result in last visit was Excellent in $63 \%$, Good in $20 \%$, Fair in $8.5 \%$ and Poor in $8.5 \%$ of the patients.

\section{Discussion}

Anatomic or close anatomic rebuilding of the articular surface is the essential objective of acetabular crack medical procedure. It is significant, in any case, to stay away from complexities identified with careful introduction of the crack, as both decrease and evasion of

Confusions are most firmly identified with a definitive clinical result of acetabular breaks. It is along these lines sensible to investigate choices for obsession of acetabular breaks that permit sufficient decrease and adjustment of the articular surface while limiting the intrusiveness of the surgery [12].

\section{Imaging}

For PCS obsession, there are 2 imaging offices either ordinary fluoroscopy or electronic route framework. As regular fluoroscopy gives a uniplanar see, numerous pictures are expected to evaluate the precision of position of the guide wire and the screw which will build the screw inclusion time, the fluoroscopic time presentation (102.2 s) and blood misfortune (57 ml).

Anyway the mechanized route framework gives a multi-planar perspectives which give a $3 \mathrm{D}$ creative mind of the life structures of the pelvis, diminishing the quantity of pictures taken and this will diminish fluoroscopic time introduction (34.7s) and blood misfortune (42 ml).

However, in our examination the employable time in electronic route framework gathering (48.2 min) was longer than ordinary fluoroscopy gathering (38 $\mathrm{min}$ ), this is ascribed to Wong et al study where mean usable time was $94 \mathrm{~min}$ as certain patients in this investigation had other pelvic wounds.

\section{Fluoroscopy time presentation}

Decrease in presentation to radiation is turning into a significant point for muscular specialists today as introduction to these radiations increment rate of skin tumors and myeloid malignancies [26]. One moment of fluoroscopy time about the pelvis is proportional to 40 $\mathrm{mSv}$ of radiation, which is what might be compared to around 250 chest radiographs. The patient ingests the majority of this radiation, albeit some is reflected to the 
specialist [27].

In our examination, the modernized route framework decline fluoroscopic time presentation more than ordinary fluoroscopy from (102.2 s to $34.7 \mathrm{~s})$.

\section{Nature of decrease}

In our examination, the mean pre-employable crack removal was $5.87 \mathrm{~mm}$. Non-dislodged and gentle uprooted $(<2 \mathrm{ml})$ breaks were fixed straightforwardly without shut decrease as happened in 51 cracks $(28 \%)$ of around 182 cracks. Uprooted cracks $(>2 \mathrm{ml}$ ) were decreased before obsession first through a shut methodology which succeded to lessen 90 breaks (49.5\%) while neglected to diminish 41 breaks $(22.5 \%)$ where smaller than usual open methodology with the guide of a decrease clasp utilized. After obsession, the mean postoperative relocation became $1.84 \mathrm{~mm}$ which is close anatomical as per Matta measures of crack decrease additionally the mean pre-usable break step was $2.17 \mathrm{~mm}$ which become $0.9 \mathrm{~mm}$ post-usable. Hong et al had preoperative hole $10 \mathrm{~mm}$ which improved to $3 \mathrm{~mm}$ postoperative by shut and smaller than normal open methods. Additionally as per Matta rules for post-usable radiological result, decrease was anatomical in $72 \%$ of our patients.

For this, shut and smaller than normal open procedures are helpful strategies for decrease of dislodged breaks agreeable for decrease by these methods.

\section{Strategy of obsession}

After break decrease, cracks were fixed either by Antegrade procedure through (AIIS) or retrograde through (pubic tubercle). The two procedures were disputable, however Frank et al revealed that occasionally antegrade method was hard to do, so a retrograde wire situation was utilized for antegrade screw strategy.

Likewise screw size was begging to be proven wrong, where either 6.5 or 7.3 somewhat strung screws were utilized.

\section{Blood misfortune and employable time}

In our examination for PCS obsession, the mean blood misfortune was $54.3 \mathrm{ml}$ going from $10 \mathrm{ml}$ announced by Lin et al to $94 \mathrm{ml}$ revealed by Wong et al. Conversely, Wu et al [28], Wong et al [29] and Daurka et al [30] announced that the mean blood misfortune in foremost methodologies for ORIF of acetabular cracks was $864 \mathrm{ml}, 782 \mathrm{ml}$ and $831 \mathrm{ml}$ individually.

Additionally in our examination for PCS obsession, the mean usable time was $43.6 \mathrm{~min}$. Interestingly, Wu et al, Wong et al and Daurka et al detailed that the mean employable time in foremost methodologies for ORIF of acetabular cracks was $220 \mathrm{~min}, 194 \mathrm{~min}$ and $233 \mathrm{~min}$ individually.

Thus, PCS obsession can decrease blood misfortune and employable time altogether contrasted with ORIF. This distinction in blood misfortune is ascribed to broad presentation and delayed usable time.
It ought to be thought about that in customary fluoroscopy bunch blood misfortune was $57 \mathrm{ml}$ however in automated route bunch blood misfortune was $42 \mathrm{ml}$, this is ascribed to the exactness and multi-planar pictures of the mechanized route framework.

\section{intricacies}

In around 430 patients with acetabular breaks just 25 patients $(5.8 \%)$ had careful complexities. Where 2 patients $(0.5 \%)$ had post-usable profound careful contaminations which were treated by debridement and IV anti-toxins, 1 patient $(0.2 \%)$ had transient femoral paralysis which was ascribed to decrease clip inclusion as opposed to screw obsession itself and settled 2 months after the activity.

5 patients (1.2\%) had intraoperative screw infiltration, just Wong et al detailed that one AC screw had intra-articular entrance yet $\mathrm{He}$ et al announced that 2 AC screws entered out of the foremost cortex of the AC in the regular fluoroscopy bunch with no related confusions watched and El-ashab et al revealed that 2 PC screws entered negligibly the average cortex without any dangers to the patient.

8 patients (1.9\%) had screw back-out, Wong et al announced that screw back-out was found in 7 patients and was noted on radiographs at a mean of 3.8 weeks with no movement of relocation in the period that followed. The mean back-out separation was $8.3 \mathrm{~mm}$ and beside a tangible noticeable quality underneath the skin in one patient, it was not related with torment or impingement side effects. Qoreishi et al detailed that screw back-out happened in one patient which was eliminated and the break proceeded to the association by weight-bearing limitation.

Wong et al ascribed this to the shirking of over-fixing of the incompletely strung screws to impact between fragmentary pressure due to osteoporotic bone. This might have allowed some level of crack dynamization and created what seemed, by all accounts, to be radiological proof of screw back-out or releasing. (24)

5 patients $(1.2 \%)$ had lost decrease, 3 in the prompt postoperative period ( 2 patients strolled with no assistive gadget in the quick postoperative period and 1 patient dropped up on the fifth postoperative day). The other two were mobile utilizing a walker when their breaks dislodged. The mean age in the loss of obsession bunch was 79 years.

1 patient $(0.2 \%)$ had break non-association in iliac peak not $\mathrm{AC}$, Wong et al detailed that this patient likewise had various back-out screws because of serious osteoporosis. Ernstberger et al revealed 3 patients (0.7\%) with obscure careful confusions.

No patient endured neural (obturator $\mathrm{n}$. furthermore, lat cut. nerve of the thigh) or vascular or instinctive injury identified with the method either intraoperatively or postoperatively. None of the patients had any huge blood misfortune. No proof of heterotopic hardening was found in any patient at the most recent development.

Then again, Shazar et al (31) revealed that careful entanglements were recorded for 31 intricacies (13.8\%) 
in around 225 patients who performed foremost pelvic methodologies. The most incessant prompt postoperative entanglement was careful site contamination requiring debridement (17 patients, 7.6\%). Every one of the 17 patients recuperated after careful debridement and a 6week course of intravenous anti-infection agents. 6 patients had nerve paralysis where 5 patients had transient Peroneal nerve earlier medical procedure and 1 patient had femoral nerve shortcoming after medical procedure. All patients recuperated 1 year after medical procedure. 1 patient had an aspiratory embolism, 2 patients had a significant prompt postoperative dying (20 units of stuffed red platelet), 2 patients had femoral course apoplexy and 1 patient had iatrogenic injury in the femoral vein. 1 patient had lost decrease during the initial 1 year of development and 1 patient had broken guidewire of the cannulated screws.

Likewise Yao et al (32) detailed that perioperative complexities were recorded for 10 difficulties (18.3\%) in around 60 patients who performed foremost pelvic methodologies. 3 patients $(5 \%)$ had delicate tissue disease. 3 patients $(5 \%)$ had postoperative profound vein apoplexy (DVT) and the outside iliac vein slashed in 1 patient $(1.7 \%)$ during the medical procedure and it was stitched quickly without apoplexy or discharge happened after activity. 3 patients (5\%) had parallel femoral cutaneous nerve injury and 1 patient $(1.7 \%)$ had obturator nerve paralysis, they all recuperated in 3 to a year after medical procedure

\section{Postoperative weight bearing}

In our investigation, all papers supported early assembly and weight-bearing where PWB with the guide of props or a walker in the first post-usable day was energized by 6 creators and full weight-bearing permitted in 4 to about two months while Kazemi empowered prompt full weight-bearing. This early weight-bearing is ascribed to the high inflexibility of intramedullary obsession.

Interestingly for ORIF strategy, Archdeacon et al [33] and Helfet et al [34] urged to utilize a touch-toe weight-bearing walk with assistive gadgets and it was plainly perceived that fractional weight-bearing was troublesome.

\section{Utilitarian result}

The utilitarian result was surveyed either by Merle d'Aubigne'scale or HHS.

As indicated by Merle, in this investigation $40.2 \%$ of patients had phenomenal result, $46 \%$ great, $5.9 \%$ reasonable and $7.9 \%$ poor, conversely Daurka et al [30] detailed that in ORIF bunch $53.4 \%$ of patients had incredible result, $35 \%$ great, $8.3 \%$ reasonable and $3.3 \%$ poor. Likewise in this examination, Kazemi et al [18] announced that the mean changed Merle d'Aubigne Score was 17.4, interestingly Elmadag et al [35] detailed that $\mathrm{I}$ in $40.9 \%$ and Poor in $13.9 \%$ of the patients.

Whereas Matta's radiological score in last visit of follow-up was Excellent in $63 \%$, Good in $20 \%$, Fair in $8.5 \%$ and Poor in $8.5 \%$ of the patients, in contrast Soni et al [36] reported that in ORIF group, Matta's radiological score in last visit was Excellent in $64.3 \%$, Good in $20 \%$, Fair in $8.6 \%$ and Poor in $7.1 \%$ of the patients also Cole et $\mathrm{al}^{(37)}$ reported that in ORIF group, Matta's radiological score in last visit was Excellent in $64 \%$, Good in $25 \%$, Fair in $7 \%$ and Poor in $4 \%$ of the patients.

So, PCS fixation is a convenient method for fracture fixation with regard to ORIF as it has a better postoperative radiological outcome and a nearly similar results for Matta's radiological score during follow-up.

\section{Conclusion}

PCS obsession of cracks of the front segment of the hip bone socket with either customary or electronic route framework fluoroscopy could be applied not exclusively to non-uprooted breaks yet additionally to dislodged cracks amiable to shut or restricted open decrease.

The modernized route framework is better as it gives a multi-planar perspectives which give a $3 \mathrm{D}$ creative mind of the life structures of the pelvis, diminishing the quantity of pictures taken and this will diminish fluoroscopic time presentation and blood misfortune.

Contrasted with ORIF, PCS obsession accomplishes a comparable nature of decrease and utilitarian result yet is related with less inconveniences, littler intraoperative blood misfortune, shorter usable time and permit early weight-bearing and rehabilitation.

\section{References}

[1] A.Laird, J.F Keating. Acetabular fractures: a 16-year prospective epidemiological study. J Bone Joint Surg (Br), Vol.87 (7), PP.969-973,2005.

[2] D.C. Mears. Surgical treatment of acetabular fractures in elderly patients with osteoporotic bone. J Am Acad Orthop Surg, Vol.7, pp.128-41,1999.

[3] T. Ferguson, R. Patel, M. Bhandari, J.M. Matta. Fractures of the acetabulum in patients aged 60 years and older: an epidemiological and radiological study. J Bone Joint Surg [Br], Vol.92-B, pp.250 257,2010.

[4] R.Judet, J.Judet, A.Lanzetta, E.Letournel, Fractures of the acetabulum: classification and guiding rules for open reduction. Arch Orthop, Vol.81(3), pp.119 158,1968 .

[5] E. Letournel, Acetabulum fractures: classifcation and management. Clin Orthop Relat Res, Vol.151, pp.81-106,1980.

[6] J.M. Matta, L.M. Anderson, H.C. Epstein, Fractures of the acetabulum. A retrospective analysis. Clin Orthop Relat Res, Vol. 205, pp.230-240,1986.

[7] d.C. Mears, J.h. Velyvis, C.P. Chang,Displaced acetabular fractures managed operatively: indicators of outcome. Clin Orthop Relat Res, Vol.407, pp.173-186,2003.

[8] J.K. Chang, S.S. Gill, R.D. Zura, Comparative strength of three methods of fxation of transverse acetabular fractures. Clin Orthop Relat Res, Vol. 392, pp.433-441,2001.

[9] P.V.Giannoudis, M.r. Grotz, C.Papakostidis, Operative treatment of displaced fractures of the 
acetabulum. A meta-analysis. J Bone Joint Surg [Br], Vol. 87-B, pp.2-9,2005.

[10] Y.C. Lin, C.h. Chen, h.t. Huang, Percutaneous antegrade screwing for anterior column fracture of acetabulum with fluoroscopic-based computerized navigation. ArchOrthop Trauma Surg, Vol. 128, pp.223-226,2008.

[11]P.Bates, A.Starr, C.Reinert, The Percutaneous Treatment of Pelvic and Acetabular Fractures. Dallas, TX: Independent Publisher (IBSN, Vol. 9781, pp.4507-3105-8); 2010.

[12]C.Adam, M.D. Crowl, Closed Reduction and Percutaneous Fixation of Anterior Column Acetabular Fractures; Computer Aided Surgery, Vol. 7, pp.169-178,2002.

[13]E. Mouhsine, R. Garofalo, O. Borens, Percutaneous retrograde screwing for stabilisation of acetabular fractures. Injury, Vol.36(11), pp.1330-1336,2005.

[14] Y.C. Lin, C.H. Chen, H.T. Huang, Percutaneous antegrade screwing for anterior column fracture of acetabulum with fluoroscopic-based computerized navigation. Arch Orthop Trauma Surg, Vol.128(2), pp.223-226,2008.

[15] M.Frank, T. Dedek, J.Trlica, J.Folvarský, Perkutánní osteosyntéza predního pilíre acetabula: první zkusenosti [Percutaneous fixation of anterior column acetabular fractures--first experience]. Acta Chir Orthop Traumatol Cech, Vol.77(2), pp.99-104,2010.

[16] G.Hong, L.Cong-Feng, H.Cheng-Fang, Z.ChangQing, Percutaneous screw fixation of acetabular fractures with 2D fluoroscopy-based computerized navigation. Arch Orthop Trauma Surg, Vol.130(9), pp.1177-1183,2010.

[17] J.L. Gary, M.VanHal, S.D. Gibbons, Functional outcomes in elderly patients with acetabular fractures treated with minimally invasive reduction and percutaneous fixation. J Orthop Trauma, Vol.26(5), pp.278-283,2012.

[18]N. Kazemi, M.T.Archdeacon, Immediate full weightbearing after percutaneous fixation of anterior column acetabulum fractures. J Orthop Trauma. vol.26(2), pp.73-79,2012.

[19]P.Schwabe, B.Altintas, K.D. Schaser, Threedimensional fluoroscopy-navigated percutaneous screw fixation of acetabular fractures. J Orthop Trauma, Vol.28(12), pp.700-706,2014.

[20] J.He, G.Tan, D.Zhou, Comparison of Isocentric CArm 3-Dimensional Navigation and Conventional Fluoroscopy for Percutaneous Retrograde Screwing for Anterior Column Fracture of Acetabulum: An Observational Study. Medicine (Baltimore), Vol.95(2), PP.e2470,2016.

[21]L.Zhang, P.Yin, W.Zhang, An Effective and Feasible Method, "Hammering Technique," for Percutaneous Fixation of Anterior Column Acetabular Fracture. Biomed Res Int, Vol. 2016, pp.7151950,2016.

[22] M.Qoreishi, H.R. Seyyed Hosseinzadeh, F.Safdari. Clinical Results of Percutaneous Fixation of Pelvic and Acetabular Fractures: A Minimally Invasive
Internal Fixation Technique. Arch Bone Jt Surg, Vol.7(3), pp.284-290,2019.

[23] G.El-ashhab Mohamed, M.El Karamany Mamdouh, M.Halawa Abdelsamie, Hassan Percutaneous Screw Fixation of Acetabular Fractures BMFJ, Vol. 36 (2), 2019.

[24] J.S.Y. Wong, J.C.K. Lau, K.H. Chui, K.L. Tiu, Three-dimensional-guided navigation percutaneous screw fixation of fragility fractures of the pelvis. J Orthop Surg (Hong Kong), Vol.27(1),2019.

[25]H.Ernstberger, P.Pieroh, A.Höch, Minimally displaced acetabulum fractures in geriatric patients: a comparison of open, percutaneous and nonoperative treatment from the German Pelvic Injury Register data. Eur J Trauma Emerg Surg,2020.

[26] M.Gundestrup, H.H.Storm, Radiation-induced acute myeloid leukaemia and other cancers in commercial jet cockpit crew: a population-based cohort study. Lancet, Vol. 354, pp.2029-2031,1999.

[27]D.M.Kahler Virtual fluoroscopy: a tool for decreasing radiation exposure during femoral intramedullary nailing. Syllabus of Fifth Annual North American Program on Computer Assisted Orthopaedic Surgery (CAOS USA), Pittsburgh, PA, July. P, PP.167-170,2001.

[28]H.Wu, L.Zhang, X.Guo, X.Jiang ,Meta-analysis of modified Stoppa approach and ilioinguinal approach in anterior pelvic ring and acetabular fractures. Medicine (Baltimore), Vol.99(4), pp.e18395,2020.

[29]X.J. Wang, L.i. Lu, Z.H. Zhang, Ilioinguinal approach versus Stoppa approach for open reduction and internal fixation in the treatment of displaced acetabular fractures: A systematic review and metaanalysis. Chin J Traumatol, Vol.20(4), pp.229234,2017.

[30] J.S. Daurka, P.S. Pastides, A. Lewis, Acetabular fractures in patients aged > 55 years: a systematic review of the literature. Bone Joint J, Vol.96-B (2), pp.157-163,2014.

[31] N.Shazar, I.Eshed, N.Ackshota, Comparison of acetabular fracture reduction quality by the ilioinguinal or the anterior intrapelvic (modified Rives-Stoppa) surgical approaches. J Orthop Trauma, Vol.28(6), pp.313-319,2014.

[32] S.Yao,K.Chen,Y.Ji, Supra-ilioinguinal versus modified Stoppa approach in the treatment of acetabular fractures: reduction quality and early clinical results of a retrospective study. J Orthop Surg Res, Vol.14(1), pp.364. Published 2019 Nov 14,2019.

[33]M.T. Archdeacon, N. Kazemi, C.Collinge, Treatment of protrusio fractures of the acetabulum in patients 70 years and older. J Orthop Trauma, Vol.27(5), pp.256-261,2013.

[34]D.L.Helfet, J.r.Borrelli J, T.DiPasquale, Stabilization of acetabular fractures in elderly patients. J Bone Joint Surg Am, Vol.74(5), pp.753$765,1992$.

[35] M.Elmadağ, Y.Güzel, M.A. Acar, G.Uzer, The 
Stoppa approach versus the ilioinguinal approach for anterior acetabular fractures: a case control study assessing blood loss complications and function outcomes. Orthop Traumatol Surg Res, Vol.100(6), pp.675-680,2014.

[36] A.Soni, R.Gupta, R.Sen ,Modified Stoppa Approach for Acetabulum Fracture: A Review. Rev Bras
Ortop (Sao Paulo), Vol.54(2), pp.109-117,2019.

[37]J.D.Cole, B.R. Bolhofner, Acetabular fracture fixation via a modified Stoppa limited intrapelvic approach. Description of operative technique and preliminary treatment results. Clin Orthop Relat Res, Vol.(305), pp.112-123,1994. 